\title{
Pattern Formation During Nanowear of Gold Films
}

Megan E. Pendergast ${ }^{1}$, Alex A. Volinsky ${ }^{1}$, and Xiaolu Pang ${ }^{1,2}$

${ }^{1}$ Department of Mechanical Engineering, University of South Florida, 4202 E. Fowler Ave. ENB118, Tampa, FL, 33620

${ }^{2}$ Department of Materials Physics, University of Science and Technology Beijing, 30 Xueyuan Rd, Haidian District, Beijing, Beijing, 100083, China, People's Republic of

\begin{abstract}
The effects of water on the wear resistance of $3 \mu \mathrm{m}$ thick sputtered gold films on silicon substrate using contact AFM and a scanning nanoindenter was investigated. In performing wear tests on gold samples in the presence of water, a significant increase in depth of the wear area was observed compared to the same tests performed in the ambient atmosphere $(\sim 55 \%$ humidity). These results were obtained using Hysitron Triboindenter on areas of $10 \times 10 \mu \mathrm{m}^{2}$ and an Atomic Force Microscope on areas of $1 \mathrm{x} 1 \mu \mathrm{m}^{2}$. Nanowear tests were preformed using silicon nitride cantilevers on the AFM and diamond Berkovich or blunt conical tips on the Hysitron. Normal loads used were $2 \mu \mathrm{N}$ and $10 \mu \mathrm{N}$, respectively. Tests performed in the ambient atmosphere resulted in a slightly reduced surface roughness, while a much higher wear rate was observed in the wear tests performed in water. Additionally, gold surface ripples formed under certain scanning conditions, in water for the Hysitron Triboindenter and in ambient atmosphere for the AFM. Nanoscale stick slip is being investigated as a possible explanation to the rippling phenomenon, and single scan line tests provide valuable information about the mechanisms and progression of the nanoscale wear.
\end{abstract}

\section{INTRODUCTION}

Wear testing is performed for various reasons, some simply to obtain an idea of longevity, and others to compare materials and different working environments [1]. Gold is used in applications ranging from dentistry to electrical connections, so it naturally evolves that the gold's response to wear is of vital importance for design [2]. Gold is often deposited in the form of a thin film for economic reasons, and sometimes the thickness is only several nanometers, exacerbating the need to study the wear properties in different environments.

Wear progression has been shown to change when comparing results at the macro vs. the nanoscale, and as such requires new explanations. Bulk material physics no longer dictates material reactions, since with the increase of the surface to volume ratio, other forces, such as surface and capillary forces affect materials response [3].

In a typical wear experiment a hard material is scanned over the tested material surface, resulting in a wear rate measurement in terms of the removed material depth as a function of normal applied load and number of wear cycles. Wear experiments performed with Hysitron and AFM are important in adding information to the "scaling down" problem in tribology because of their own relative size differences. Hysitron tests were preformed on $10 \times 10 \mu \mathrm{m}^{2}$ areas, while AFM tests were performed on $1 \times 1 \mu \mathrm{m}^{2}$ areas.

The field of nanoscale tribological testing, more specifically with AFM is still in its very early stages in terms of understanding and being able to quantify certain aspects such as tip 
characterization and force calibration $[4,5]$. These processes are not yet standardized so the field of nanotribology still remains largely unresolved.

It has been shown that AFM experiments in varying humidity have displayed significant increases in friction [6]. Fortunately, gold does not chemically react with water, so chemical effects on the wear progression can be ruled out. In experiments performed on PVD sputtered gold in AFM, an increase in friction could be seen with increasing humidity. The authors suggested that capillary forces are adding to this friction coefficient in the presence of water [6]. It is important to note, that no increase in friction was seen after increasing humidity above $33 \%$. Gold, being hydrophilic in nature, could have already reached its saturation point, therefore any increase in humidity would not affect the frictional values. Other experiments showed that to increase the frictional values in AFM scanning, it is essential that the humidity be high enough for the surface material to support at least a monolayer of water [7].

As the friction coefficient changes during experiments in varying humidity, the wear progression can also be affected. The wear mechanism can evolve differently, as seen in polymer wear tests [8]. PEEK matrix polymer composites showed abrasive wear in a dry environment, while erosive wear was present in water wear experiments.

Wear testing has also been shown to produce interesting surface patterns, as ripples have been observed in wear testing of $\mathrm{KBr}$, and single crystal Aluminum $[9,10]$. This texturing of the surface can have important implications as nanostructures and patterns are influencing drug delivery, studying lubrication channels, and solving debilitating issues like stiction in MEMS devices. Surface modification and characterization is a rapidly emerging field at the nanoscale because of the instruments available to modify, as well as characterize surfaces.

\section{EXPERIMENTAL DETAILS}

Sputtered gold films, $3 \mu \mathrm{m}$ thick were cleaned with acetone and methanol before conducting AFM and Hysitron wear tests. The Hysitron experiments were preformed with a conical diamond tip ( $1 \mu \mathrm{m}$ tip radius, machine compliance of $3 \mathrm{~nm} / \mathrm{N}$ ). The AFM tip was a silicon nitride cantilever with a radius of $100 \mathrm{~nm}$ and $0.2 \mathrm{~N} / \mathrm{m}$ spring constant. The Hysitron experiments were performed with a normal load of $10 \mu \mathrm{N}$, while the AFM experiments were performed at $2 \mu \mathrm{N}$. Test parameters were kept the same when testing in ambient atmosphere (55\% humidity) or in a distilled water environment. The test areas were 10x10 $\mu \mathrm{m}^{2}$ and $1 \times 1 \mu \mathrm{m}^{2}$ in the Hysitron and AFM, respectively.

\section{DISCUSSION}

Figure 1 shows results of wear testing in the ambient environment in the AFM. From the topography and cross section of the gold surface topography, it is apparent that there is minimal wear, on the order of $5 \mathrm{~nm}$. Surface modification or smoothing of asperities appears in the ambient experiments. Results obtained in the Hysitron Triboindenter also displayed minimal wear on the order of approximately $15 \mathrm{~nm}$. 

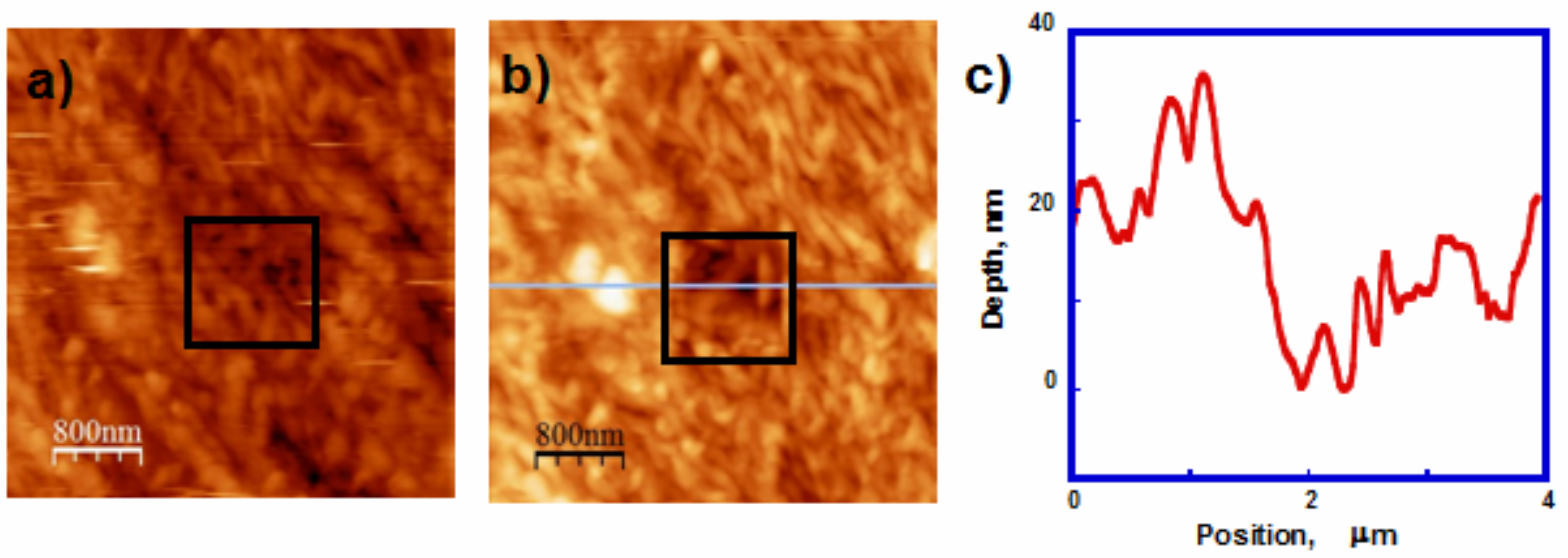

Figure 1. Sputtered gold film tested in ambient environment in AFM at $2 \mu \mathrm{N}$ normal load, $10 \mathrm{~Hz}, 1 \times 1 \stackrel{\mu}{\mathrm{m}}$ scan area: a) Initial surface with a square outlining the wear test area; b) Surface after 30 scans with the same identifying square; c) Cross-section showing depth reached in the scan area. Both AFM and Hysitron images are made up of 256 scan lines per image.

Figure 2 shows the results of the wear tests performed in the presence of water. In the AFM experiments the test was performed for the same number of scans and produced a depth of 20 times greater than in the ambient experiments. Experiments performed in the Hysitron Tribolindenter were performed for half the number of scans as the ambient experiment, and similarly to AFM results, produced 10 times higher wear rate.
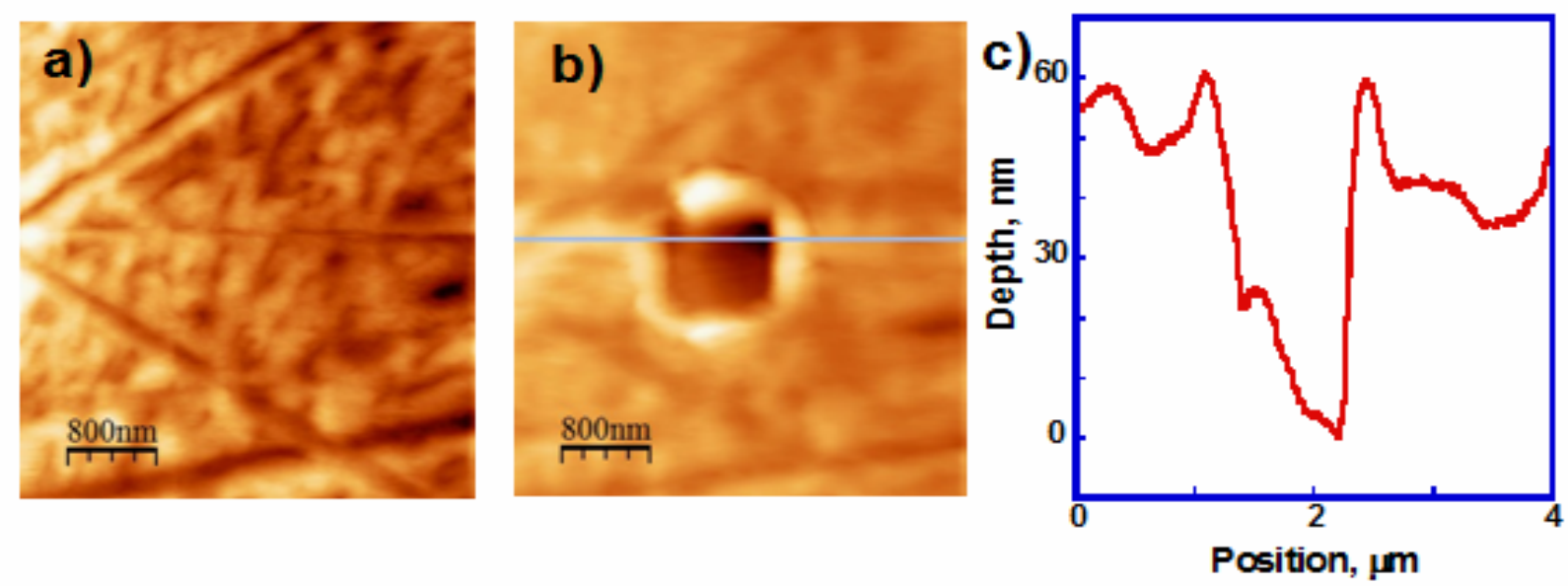

Figure 2. Sputtered gold film on silicon scanned over a 1x1 $\mu \mathrm{m}^{2}$ area in AFM for 30 scans at $10 \mathrm{~Hz}, 2 \mu \mathrm{N}$ normal load in the presence of water: a) Initial surface; b) Surface after 30 scans; c) Cross-section showing depth reached in the scan area.

Another interesting phenomenon that was observed in both tools in different conditions was the presence of ripples that formed during scanning. Figure 3 shows these ripples that appeared in the ambient environment in the AFM after only 30 scans of the gold surface. Figure 4 shows the ripples observed in water using the Hysitron. The ripples are aligned perpendicular to the long scan direction, and the gold pile up can be seen clearly in Figure 4 around the edges of the wear track, indicating the possibility of more plastic deformation than actual material removal due to wear. Similar surface rippling has been observed on the $\mathrm{KBr}$ surface in air. 

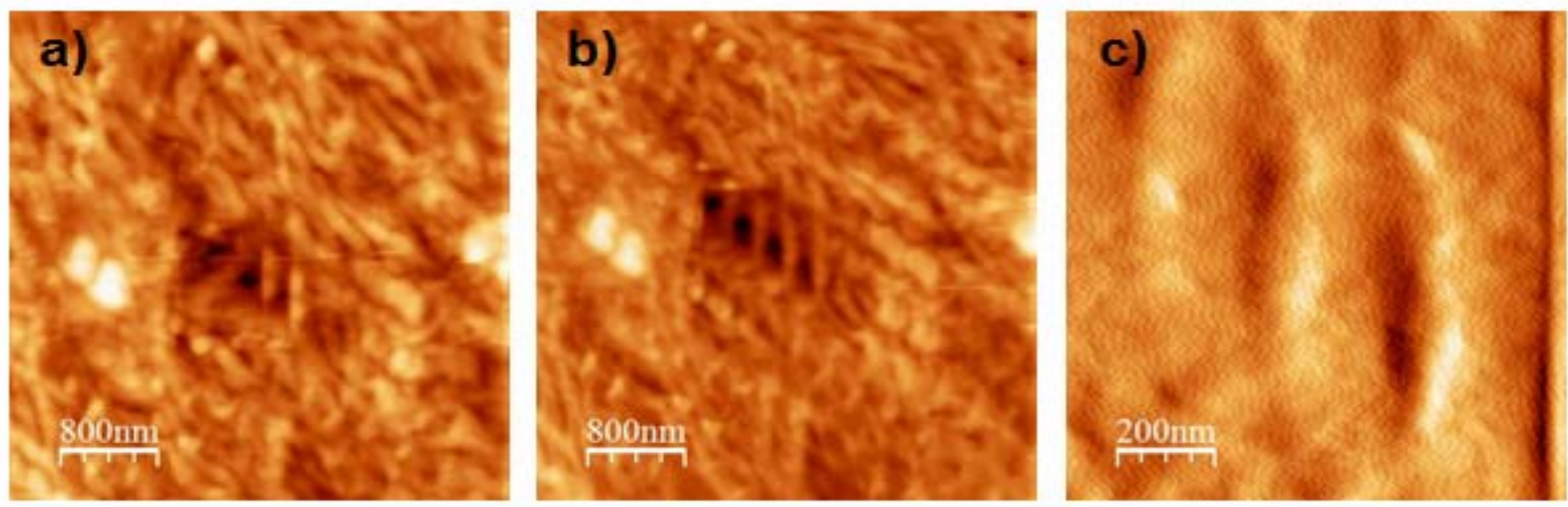

Figure 3. Sputtered gold, $2 \mu \mathrm{N}$ normal load, ambient conditions after: a) 30 scans; b) 50 scans; c) 50 scans, $1 \times 1 \mu \mathrm{m}^{2}$ AFM deflection image.
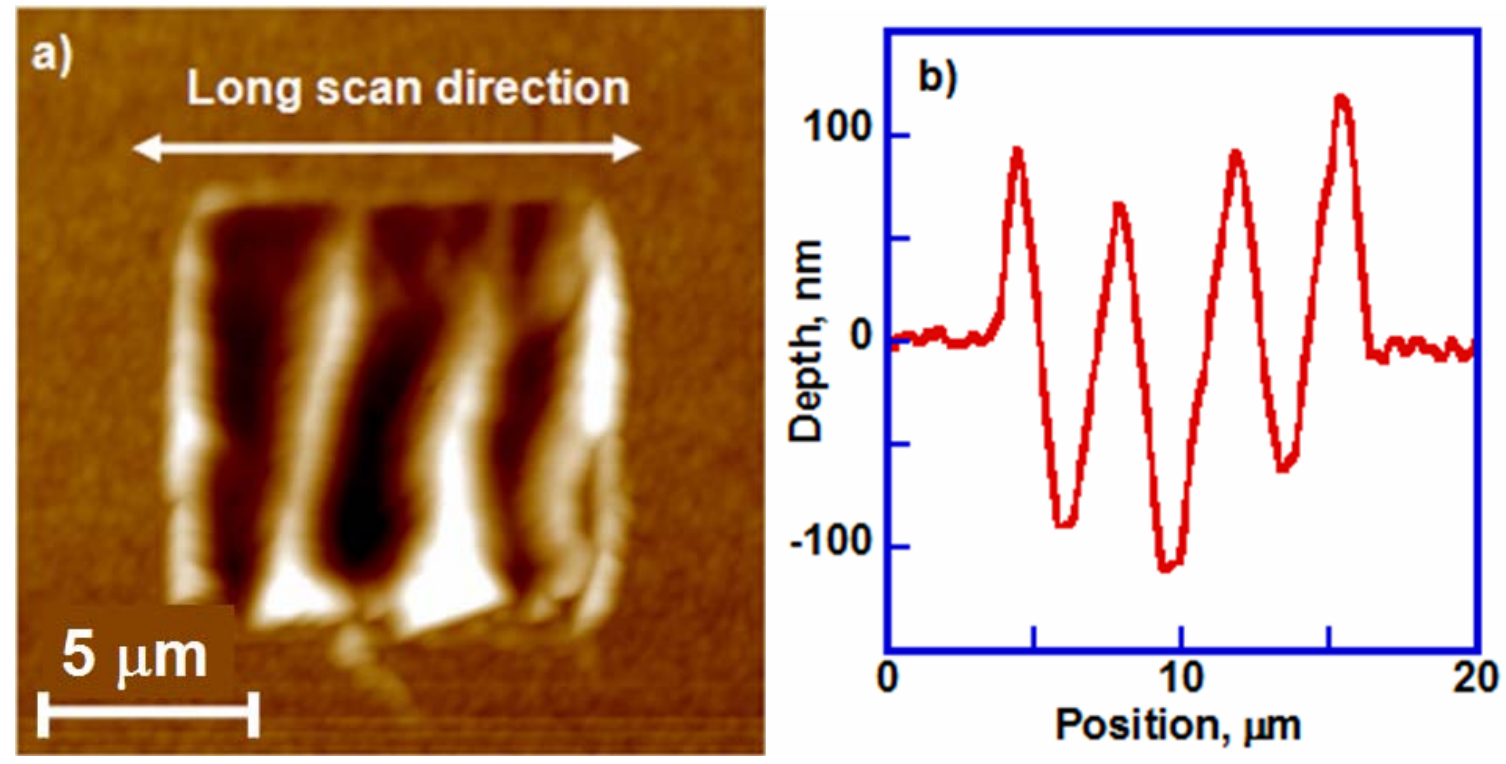

Figure 4. Wear pattern and b) Wear track of a gold film after 200 wear cycles at $10 \mu \mathrm{N}$ normal load with a sharp Berkovich tip in water showing surface ripples.

This drastic increase in material removal observed in the experiments performed in the presence of water could possibly be due to moisture-assisted grains fracture and pull-off, with more material going into solution and increased friction. An additional test preformed was scanning a single line repeatedly in the AFM to obtain an idea of the wear rate corresponding with depth. An example of this can be seen in Figure 5b. The depth of scan lines (Figure 5) plateaus at a certain depth even when the number of line scans increases. With constant normal load the tip contact area is increasing during abrasive wear, until the contact pressure is reduced below the level of gold hardness, at which point the wear process transitions from abrasive to frictional wear. 
The ripples observed share a similar pattern to stick slip motion. As the kinetic coefficient of friction is most always lower than the static coefficient, it is rare that movement will be perfectly smooth; rather, some "jumpiness" is to be expected. Every object has some degree of elasticity, including indenter and AFM tips. Usually this elasticity causes a degree of stick slip motion that is small enough that it is essentially unnoticeable, but still exists on a reduced scale [11].

In many poorly designed sliding instances, pile up is produced ahead of the motion, thus increasing the friction. This continues to increase until a point of instability is reached and sliding occurs at the lower original friction value. Something must yield to cause this drop in friction, and this type of motion is called "spragging" $[12,13]$. In the ripples observed, the tip could plastically deform (ripple) the gold, then yield (spragging) and repeat the process forming the ripples on both the micro and nano scales.
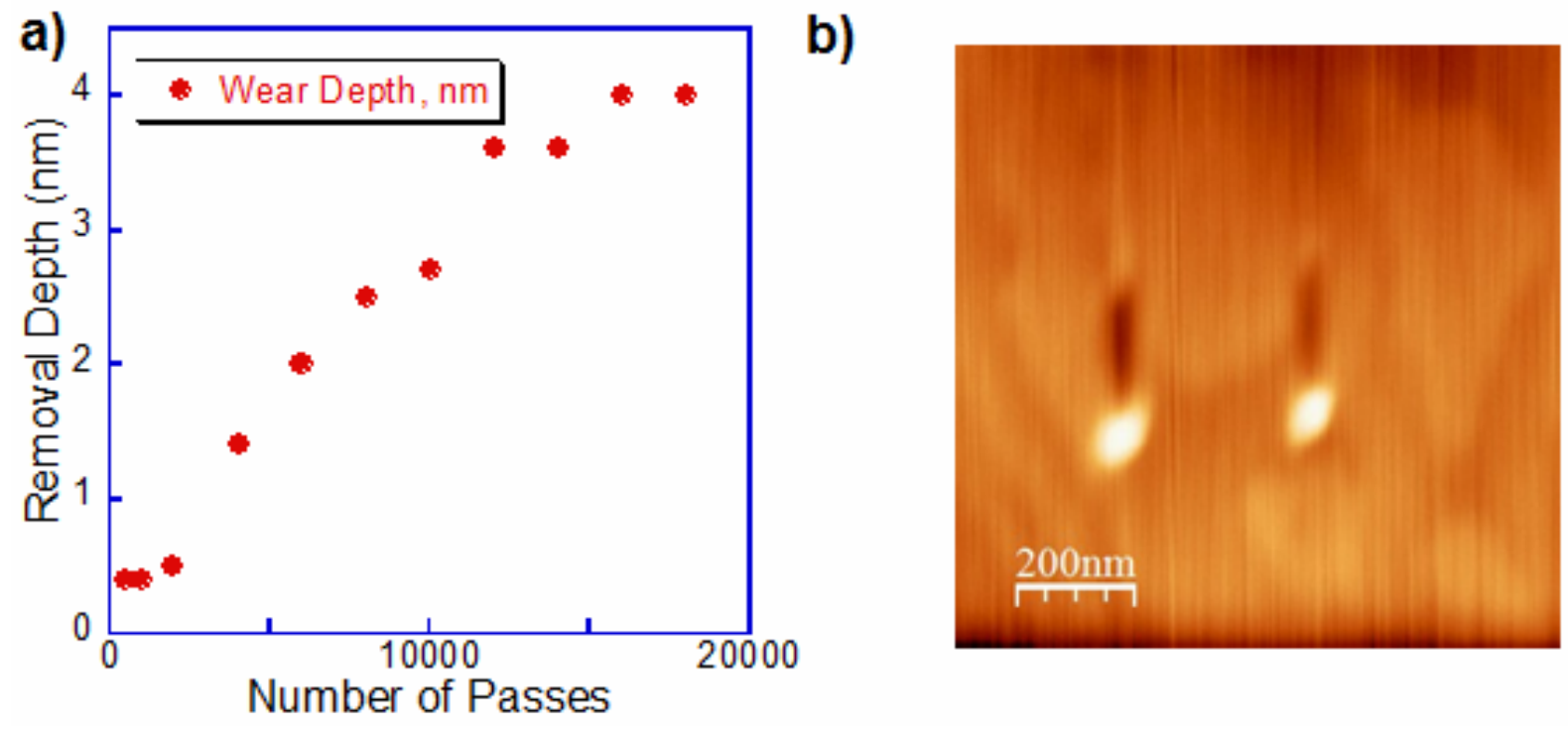

Figure 5. Single scan line wear data portraying the "plateau effect" after initial rapid wear and b) An example of the single scan line wear performed on the single crystal gold.

\section{CONCLUSIONS}

The addition of water to a wear experiment on gold produced considerably deeper wear areas than its ambient counterpart. Topography modification appears to be the main mechanism of ambient wear tests at the nanoscale, whereas much higher wear rate and nanoripples are observed in water. Moisture is influencing the material removal with increased wear rate. Most likely this moisture is assisting grain fracture and pull-off.

The ripples formed on gold were not just a phenomenon attributed to one material, as they have been observed in $\mathrm{KBr}$, aluminum and calcite single crystals [10]. Mechanisms responsible for rippling, including system dynamic response and stick slip behavior are being investigated. 


\section{ACKNOWLEGDEMENTS}

This work is supported by the National Science Foundation (CMMI-0631526), the division of Design, Manufacture and Industrial Innovation, and the Marie Curie Host Fellowship for the Transfer of Knowledge under the project "Nano-Engineering for Expertise and Development - NEED" within the $6^{\text {th }}$ EU Framework Program. The authors would also like to thank Prof. Marek Szymonsli's group for collaboration in this project, Oden Warren and Lance Kuhn from Hysitron Inc. for valuable discussions.

\section{REFERENCES}

1. J. Celis, Surface and Coatings Technology, 74-75 pp.15-22, (1995).

2. J. Barriga, B. Fernandez-Diaz., A. Juarros, S.I.-U. Ahmed, J.L. Arana, Tribology International 40, pp. 1526-1530 (2007).

3. R. Carpick, Science 313, pp. 184-185 (2006).

4. A. Socoliuc, E. Gnecco, R. Bennewitz, E. Meyer Physical Review B 68 (2003).

5. $\quad$ R. Carpick, M. Salmeron, Chem. Rev. 91 pp. 1163-1194 (1997).

6. R.G. Chadwick, H.L. Mitchell, I. Cameron, B. Hunter, M. Tulley, Journal of Dentistry 25 pp. 41-47 (1997).

7. L. Xu, H. Bluhm, M. Salmeron, Surface Science 407 pp. 251 - 255 (1998).

8. R. Prehn, F. Haupert, K. Friedrich Wear 259 pp. 693-696 (2005).

9. M. Pendergast, A. Volinsky, R. Gunda, MRS Proceedings (2007).

10. E. Gnecco, R. Bennewitz, A. Socoliuc, E. Meyer, Wear 254 pp.859-862 (2003).

11. P. Bowden, D. Tabor, "Friction, an introduction to tribology", Krieger Publishing, (1982).

12. D. Karnopp, Transactions of the ASME, 107 (1985).

13. B. Armstrong-Helouvry, P. Dupont, C. Canudas De Wit, Automatica, 307 pp. 10831994, (1994). 